noyée dans le béton du mur de garde. On avait, en outre, jeté dans le lit de la rivière, en amont de l'ouvrage, de la cendre volcanique très fine, trouvée dans le volsinage, qui vint se déposer au pied du barrage sur une épaisseur de descendu jusqu'à la couche imperméable. Et mèmo, si le mur de garde devait être descendu très profond, il serait bon de prolonger sous la dalle de fondation quelques-uns des murs de contreforts intérieurs de manière à former

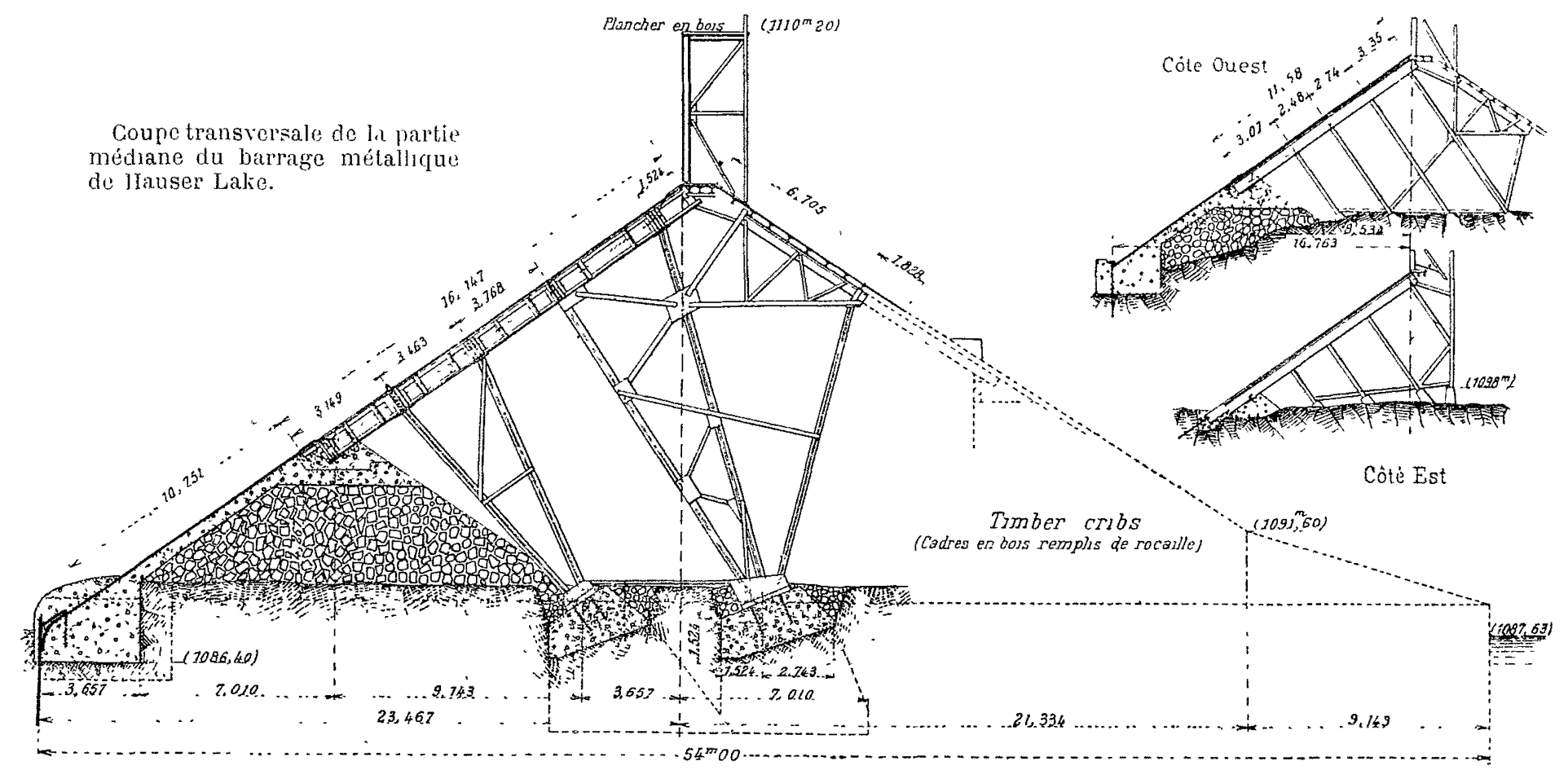

$6 \mathrm{~m}$., et s'étendait jusqu'à $300 \mathrm{~m}$. à l'amont. Malgré toutes ces précautions, une partie du barrage a été emporté après quelques mois seulement de service.

La rupture s'est produite lorsque le réservoir était plein. Des affouillements se produisirent sou pierres sèches qui s'aflaissa. Le platelage en tôle n'étant plus suffisamment supporté, l'un des joints de dilatation ne tarda pas à se rompre, provoquant ainsi une brèche dans le parement amont. Sous la poussée de l'eau qui s'y précipita, les affouillements prirent immédiatement une importance telle que les fondations des fiches d'appui cédèrent à leur tour, provoquant la rupture du barrage qui s'ouvrit complètement sur $90 \mathrm{~m}$. de largeur, et se replia parallèlement aux rives.

Comme le Missouri coule sur $45 \mathrm{kms}$ à l'aval du barrage dans des gorges presque inhabitées, les dommages causés par la rupture de cet ouvrage furent relativement peu élevés, et ne sont estimés qu'à 300000 dollars. Il n'y a pas elı mort d'homme.

L'usine du Hauser Lake était disposée immédiatement à l'aval du barrage, perpendiculairement à celui-ci. Elle n'a presque pas souffert. Elle contenait 5 groupes électrogénes de $4000 \mathrm{HP}$ char,un, produisant du courant triphasé qui était transmis à 70000 volts aux lieux de consommation, concuremment avec celui produit par une autre usine de la même Compagnie, située à Ferry Canyon, à $26 \mathrm{kms}$ en amont.

Les extrémités de l'ouvrage, qui avaient été fondées sur le rocher, ayant bien résisté, les ingénieurs de la Helena Power $\mathrm{C}^{\circ}$ songeaient quand même, parait-il, quelque temps après la catastrophe, à reconstruire la partie rompue du barrage d'après le même système. Nous ne savons s'ils ont persisté dans leur intention, mais nous n'avons, quant à nọus, aucune confiance sur l'efficacité de cette réparation.

Pour un barrage comme celui-ci, devant reposer, en partie, sur un terrain graveleux, nous preférerions de beaucoup un barrage creux, en ciment armé, analogue à celui de l'usine de Colliersville et à ceux qui ont été décrits dans les numéros de La Houille Blanche de juillet 1906 et de mars 1908, avec dalle de fondation et mur de garde des nervures destinées à renforcer ce mur de garde, et a l'empêcher de fléchir sous un excès de poussée du terrain amont,

II. BELLET.

\section{NOUVELLE THÉORIE DES TURBINES}

Par le Professeur Dr H. Lonenz, de Danzig (Suite)

\section{II. - Les turbines parallèles}

En coupe méridienne, nous considérerons le profil de la roue d'une turbine parallèle comme limité par deux horizontales $\eta_{1}$ et $\tilde{\eta}_{2}$, et deux couples de lignes d'écoulement

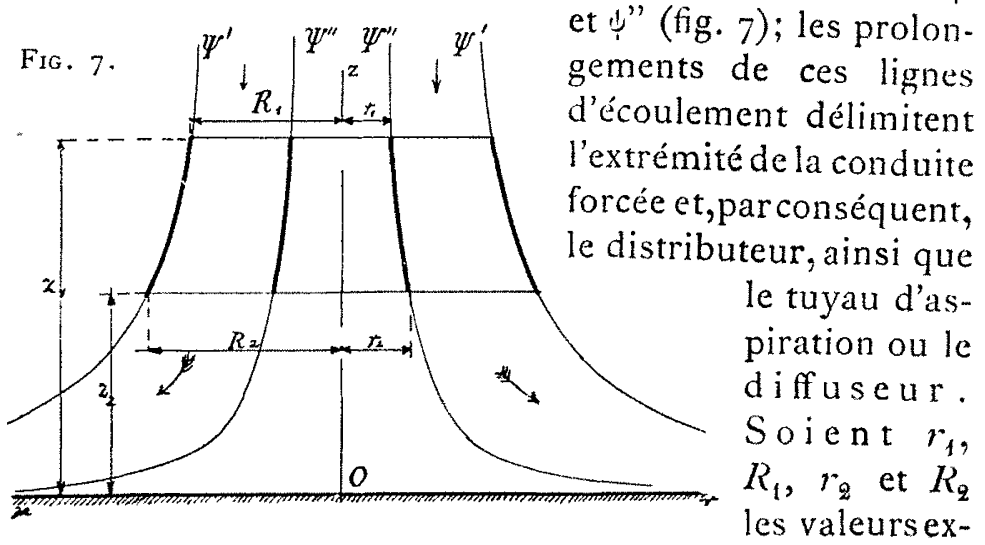
trêmes des rayons dans le plan supérieur et le plan inférieur. Le débit qui s'écoule par unité de temps est donné d'après la relation( (14) par la formule :

$$
Q=2 \pi \gamma\left(\psi^{\prime}-\psi^{\prime \prime}\right)
$$

et par conséquent, d'après la relation (21), par :

$$
Q=2 \pi \% A\left(R_{1}^{2}-r_{1}^{2}\right) z_{1}=2 \pi_{\gamma} A\left(R_{2}^{2}-r_{2}^{2}\right) \tilde{z}_{2} .
$$


Lc moment de rotation de la turbine est, d'après les relations (22):

$\mathfrak{N}=2 \pi \frac{\gamma}{g} A \iint\left[2 r z \frac{\partial\left(w_{u} r\right)}{\partial \tau}-r^{2} \frac{\partial\left(w_{u} r\right)}{\partial r}\right] \mathrm{d} r \mathrm{~d} z$.

Pour faire cette intégration, nous disposons de la valeur de $n_{u} r$. D'après le caractère de cette turbine, nous pourons faire abstraction des variations de ce produit avec $r$ et décider que tous les éléments des aubes doivent exercer sur l'eau la même accélération de réaction $q_{u}$, dans le sens de la rotation, ce qui correspond à supposer la pression uniforme sur la coupe méridienne; dans ces conditions, le terme entre parenthèses de l'équation (24) se réduit à $2 \mathrm{Br}$, $B$ étant une constante, et nous avons :

soit :

$$
\frac{\partial\left(u_{u} r\right)}{\partial r}=0 \quad \sim \frac{\partial\left(w_{u} r\right)}{\partial r}=B
$$

$$
w_{u} r=B \mathrm{~L}_{\bar{\imath}}+C=B \mathrm{~L} \frac{i}{i_{0}}
$$

$C$ et $z_{0}$ désignant des constantes d'intégration. L'équation (24) s'écrit alors :

$$
\Re=4 \pi \frac{i}{g} A B \int_{j}^{j} r \mathrm{~d} r \mathrm{~d}_{i}
$$

soit, en faisant l'untégration entre les limites $\tilde{i}_{1}$ et $\tilde{z}_{2}, R_{1}, r_{1}$ et $R_{2}, r_{2}$ :

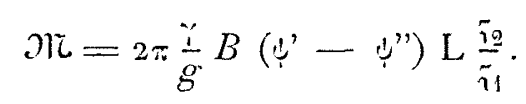

En nous reportant à la relation (23), et en remarquant que, d'apres la relation (25), nous avons:

$$
\begin{aligned}
& w_{u_{1}}{ }^{\prime} R_{1}=B \mathrm{~L} \frac{\pi_{1}}{\tau_{0}}, \quad \quad w_{u_{n}}^{\prime} R_{2}=B \mathrm{~L} \frac{z_{2}}{\tau_{0}} \\
& w_{u_{1}} " r_{1}=B L \frac{\pi_{1}}{z_{0}}, \quad w_{u_{2}}{ }^{\prime} r_{2}=B L \frac{\tau_{2}}{\tau_{0}}
\end{aligned}
$$

nous écrirons l'équation du moment sous la forme:

$\mathfrak{K}=\frac{Q}{g}\left(w_{u_{2}}{ }^{\prime} R_{2}-w^{\prime} u_{1} R_{1}\right)=\frac{Q}{g}\left(w_{u_{2}} " r_{2}-w_{u_{1}} " r_{1}\right)\left({ }_{24} c\right)$

L'équation du moment de la turbine parallèle est donc analogue à celle d'Euler, le produit w ur n'élant, d'après la relation $(25)$, fonction que de la hauteur $z$.

Pour pouvoir construire l'aubage de la turbine, nous considérerons la trajectoire absolue des particules liquides. L'équation de cette trajectoire est déterminée par les relations :

$$
\frac{\mathrm{d}_{\mathrm{i}}}{\mathrm{d} t}=w_{z}, \quad \frac{\mathrm{d} r}{\mathrm{~d} t}=w_{r}, \quad r \frac{\mathrm{d} \mathrm{i}_{\mathrm{i}}}{\mathrm{d} t}=w_{u},
$$

entre lesquelles nous éliminerons le temps $t$. Nous savons déjà que chacune des trajectoires des particules liquides se trouve sur une surface de révolution engendrée par les lignes d'écoulement; il nous suffira donc de chercher la relation qui lie l'angle $\varphi$ aux valeurs de $r$ et de $z$. En éliminant le temps entre ces dernières relations, et en tenant compte des relations $(22)$ et $(25)$, nous obtenons:

$$
r^{2} \frac{\mathrm{d}_{0}}{\mathrm{~d}_{i}}=\frac{w_{u} r}{w_{i}}=\frac{B}{2 A_{i}} \mathrm{~L} \frac{\tilde{i}}{i_{0}}
$$

soit, puisque $\downarrow=A r^{2} \bar{i}$,

$$
\mathrm{d}_{\varphi}==\frac{B}{2 !} \mathrm{L} \frac{z}{i_{0}} \mathrm{~d}
$$

Enintégrant cette expression, en posant $\varphi=$ o pour $z=z_{1}$ nous obtenons l'équation de la trajectoire absolue :

$$
i=\frac{B}{2 \downarrow}\left(2 \mathrm{~L} \frac{i}{i_{0}}-\pi_{1} \mathrm{~L} \frac{i_{1}}{i_{0}}-\tilde{i}+\tilde{i 1}_{1}\right)
$$

Pour passer de la trajectoire absolue à la trajectoire relative, nous avons:

soit :

$$
\mathrm{d} \psi=\mathrm{d} p-\omega \mathrm{d} t=\mathrm{d} \varphi-\omega \frac{\mathrm{d}_{i}}{2 w_{i}},
$$

$$
\mathrm{d} \%=\left(\frac{B}{2 !} \mathrm{L} \frac{i}{\tau_{0}}-\frac{\omega}{2 A_{\tau}}\right) \mathrm{d} \tau,
$$

ou, après intégration, en posant $\%=0$, pour $z=z_{1}$ :,

$$
x=\frac{B}{2 !}\left({ }_{i} \mathrm{~L} \frac{\pi}{z_{0}}-\pi_{1} \mathrm{~L} \frac{i}{\tau_{0}}-i+\pi_{1}\right)-\frac{\omega}{2 A} \mathrm{~L} \frac{z}{z_{1}}
$$

$\mathrm{Si}$, en outre, nous nous donnons la drection générale des aubes près de l'entrée de la roue (suivant un rayon par exemple), nous aurons par l'équation (26) la forme de l'extrémité de la directrice, et par l'équation (27) la forme de toute l'aube de la roue. Si, de plus, nous supposons un diffuseur pour conduire l'eau à sa sortie de la roue, il faudra, au voisinage de la roue, construire les aubes de ce diffuseur suivant l'équation (26), c'est-à-dire, suivant le prolongement des directrices du distributeur.

Le problème de la construction d'une turbine parallèle est ainsi complètement résolu théoriquement. Il reste á indiquer la marche des calculs à faire en pratique, en appliquant nos formules; le plus simple sera de traiter un exemple numérique.

Soit donc à construire une turbine parallele pour une hauteur de chute $h=5 \mathrm{~m}$., et un débit $Q=2 \mathrm{~m} .{ }^{3}$ par seconde.

La vitesse maxima correspondant à cette hauteur de chute est :

$$
w_{0}=\sqrt{2 g h}=9,9 \text { I mètres à la seconde. }
$$

Donnons-nous, comme valeurs des vitesses de l'eau à l'entrée et à la sortie de la roue parallèlement à l'axe :

$$
w_{\mathfrak{i}^{1}}=-2^{\mathrm{m}}, 5 \text { et } \quad w_{\mathfrak{q}^{2}}=-2 \mathrm{~m} \text {. à la seconde. }
$$

Le signe - est dû au choix de l'origine des coordonnées.

Prenons, comme hauteur de la roue, la valeur:

$$
\tilde{z}_{1}-\tilde{z}_{2}=-\mathrm{o}^{\mathrm{m}} 25 .
$$

Des relations (22) nous déduisons:

$$
2 A\left(\tilde{i 1}_{1}-\tilde{i 2}_{2}\right)=w_{i 1}-w_{12} \text {; }
$$

d'où : $\quad A=-\mathrm{I}, \quad z_{1}=\mathrm{Im}, 25, \quad z_{2}=\mathrm{I} \mathrm{m}$.

De la relation: $Q=\gamma \pi\left(R_{1}{ }^{2}-r_{1}{ }^{2}\right) w_{q^{1}}$, nous tirons : $\quad \pi\left(R_{1}{ }^{2}-r_{1}{ }^{2}\right)=0,80 \mathrm{~m}^{2}$.

Cette équation est satisfaite pour les valeurs :

$$
R_{1}=\mathrm{o}^{\mathrm{m} 6 \mathrm{o}} ; \quad r_{1}=\mathrm{o}^{\mathrm{m} 325 ;} \quad R_{1}-r_{1}=\mathrm{o}^{\mathrm{m}} 27^{5} .
$$

Nous connaissons maintenant l'ouverture de la roue à l'entrée, et le paramètre des deux courbes limites de la roue en coupe méridienne :

$$
\begin{aligned}
& \bigcup^{\prime}=A R_{1}^{2} z_{1}=-0,45 ; \\
& \bigcup^{\prime \prime}=A r_{1}^{2}{ }^{2}=-0,13 ;
\end{aligned}
$$


les rayons de sortie ont pour valeurs :

$$
\begin{aligned}
& R_{2}=\sqrt{\frac{\overline{\omega^{\prime}}}{A_{\mathrm{\imath}_{2}}}}=\mathrm{o}^{\mathrm{m} 67} ; \\
& r_{2}=\sqrt{\frac{d^{\prime \prime}}{A_{i_{2}}}}=0^{\mathrm{m} 36} \text {. }
\end{aligned}
$$

La figure 8 représente la coupe méridienne de la cou-

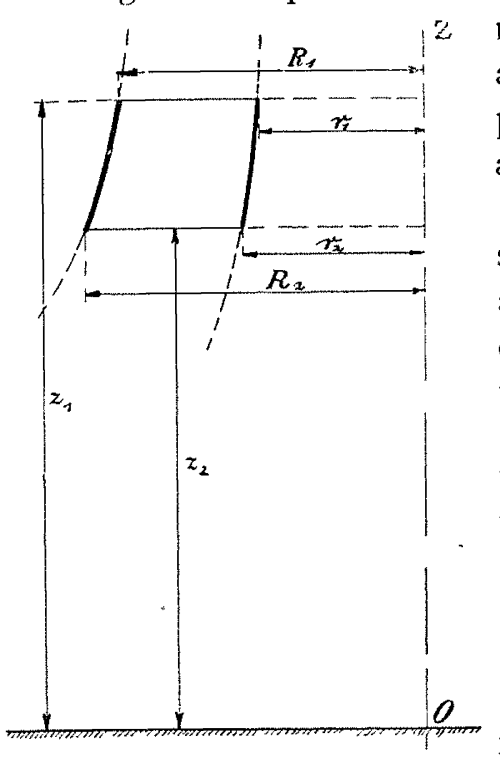
ronine de la roue mobile, avec tous ses éléments et sa position au-dessus du bief aval.

Les vitesses radiales (par seconde) aux sommets des angles de cette coupe méridienne se déduisent des relations (22):

$w_{11}^{\prime}=\mathrm{o}^{\mathrm{m} 6 \mathrm{o}}, \quad w_{r_{1}}^{\prime \prime}=\mathrm{o}^{\mathrm{m}} 325$, $w_{r_{2}}^{\prime}=\mathrm{o}^{\mathrm{m}} 6_{7}, \quad w_{r_{2}}{ }^{\prime \prime}=\mathrm{o}^{\mathrm{m} 360}$.

Ces valeurs sont toutes très faibles.

L'angle $\alpha$, donné par la Fig. 8. relation : $\operatorname{tg} \alpha=\frac{w_{\tau}}{w_{u}}$

donne d'une façon très approchée l'angle de la trajectoire absolue avec le plan horizontal (fig. 9). Par raison de construction, nous ne prendrons pas, pour les directrices, une valeur de $\alpha$ inférıeure à $20^{\circ}$; la valeur minima de $\operatorname{tg} \alpha$ sera : $\operatorname{tg} \alpha=0,364$. Cette valeur, d'après la relation $(25)$, correspond à l'entrée près de la couronne intérieure, et nous aurons en ce point :

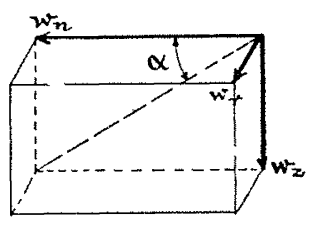

FIG. 9 .

$$
w_{u_{1}} "=\frac{w_{i 1}}{\operatorname{tg} \alpha}={ }^{\circ} 6,87 \mathrm{~m} / \mathrm{sec} .
$$

D'après la relation $(25)$, pour la même hauteur $z_{1}$, nous avons :

$$
w_{u_{1}} R_{1}=w_{u_{1}} " r_{1}=2,223 \mathrm{~m}^{2} / \mathrm{sec} .,
$$

soit, pour l'entrée près de la couronne extérieure :

$$
w^{\prime}{ }_{u_{1}}=w^{\prime \prime}{ }_{u_{1}} \frac{r_{1}}{R_{1}}=3,72 \mathrm{~m} / \mathrm{sec} .
$$

avec un angle $\alpha$ ' pour la directrice donné par :

$$
\operatorname{tg} \alpha^{\prime}=\frac{w_{31}}{w_{u_{1}}}=0,67_{2}, \quad \text { soit } \alpha^{\prime}=34^{\circ} \text {. }
$$

Nous voyons donc que l'inclinaison de la directrice sur le plan horizontal va en croissant de l'intérieur vers l'extérieur.

Supposons, en outre, que la composante tangentielle de la vitesse de l'eau à sa sortie de la roue soit nulle; d'après la relation $(25)$, nous aurons :

soit :

$$
B \mathrm{~L} \frac{z_{2}}{z_{0}}=w_{u_{2}} \dot{R}_{2}=w^{\prime}{ }^{\prime}, r_{2}=0 ;
$$

$$
\tau_{0}=\tau_{2}=1 \mathrm{~m} \text {; }
$$

et, d'après la reiation $(25 \mathrm{~b})$ :

$$
\begin{gathered}
B \mathrm{~L} \frac{\tilde{1}}{\bar{i}}=w^{\prime} R_{1}=2,233 \mathrm{~m} / \mathrm{sec} ., \\
\mathrm{L} \frac{\tilde{11}}{\pi 2}=\mathrm{L} 1,25=0,223, \\
B=10 .
\end{gathered}
$$

Le moment de rotation de la turbine a pour valeur:

$$
\begin{gathered}
\Re=-\frac{Q}{g} w_{u_{1}}^{\prime} R_{1}=-\frac{2000}{9,81} \times 2,233, \\
\Re=-455 \text { kilogrammètres. }
\end{gathered}
$$

La vitesse angulaire (1), et le nombre de tours à pleine charge, sont liés par la relation:

$$
n=\frac{30(1)}{\pi}
$$

et ont respectivement pour valeurs :

$$
\omega=-\frac{T}{\pi \mathrm{K}}=22, \mathrm{o}, \quad n=2 \mathrm{ro} .
$$

Ces valeurs supposent que la turbine travaille sans pertes, et que la vitesse de l'eau à sa sortie de la roue $\left(n w_{70}\right.$ et $\left.m_{10}\right)$ peut être abaissée dans le tuyau d'aspiration jusqu'à prendre la valeur de la vitesse de l'eau dans le canal de fuite. Mais, comme cette condition ne peut pas être remplie cn pratique, nous ferons intervenir le rendement de la turbine $r_{n}$, et nous aurons :

$$
\omega=-\frac{r_{1} T}{\mathfrak{M} T}
$$

et, en prenant : $r_{i}=0,85$, nous aurons :

$\omega=18,7$ et $n=180$ tours par minute environ.

Les vitesses tangentielles aux quatre angles de la coupe méridienne ont pour valeurs par seconde :

$$
\begin{aligned}
& u_{1}^{\prime}=R_{1} \omega=\mathrm{II} \mathrm{I}^{\mathrm{m}}, 22 ; \quad u_{1}^{\prime \prime}=r_{1} \omega=6^{\mathrm{m}}, 08 \\
& u_{2}^{\prime}=R_{2} \omega=\mathrm{I} 2^{\mathrm{m}}, 53 ; \quad u_{2}{ }^{\prime \prime}=r_{2}\left(\omega=6^{\mathrm{m}}, 73 .\right.
\end{aligned}
$$

La relation : $\quad \operatorname{tg} \beta=\frac{w_{i}}{u-w_{u}}$,

déduite de la figure io, donne l'angle que fait l'extrémité de l'aube avec l'horizontale en développement cylindrique. Nous aurons donc:

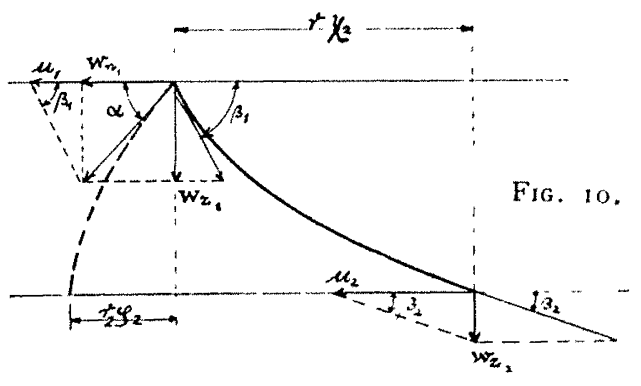

à l'entrée: $\left\{\begin{array}{l}\operatorname{tg} \beta_{1}^{\prime}=\frac{w_{31}}{u_{1}^{\prime}-w_{u_{1}}}=0,575 ; \quad \beta_{1}^{\prime}=30^{\circ} ; \\ \operatorname{tg} \beta_{1}{ }^{\prime \prime}=\frac{u w_{31}}{u_{1}^{\prime \prime}-w_{u_{1}}{ }^{\prime \prime}}=1,06 ; \quad \beta_{1}^{\prime \prime}=46^{\circ} 40^{\prime} ;\end{array}\right.$ et à la sortie : $\begin{cases}\operatorname{tg} \beta_{2}{ }^{\prime}=\frac{w_{12}}{u_{2}{ }^{\prime}}=0,159 ; & \beta_{2}^{\prime}=9^{\circ} ; \\ \operatorname{tg} \beta_{2}{ }^{\prime \prime}=\frac{w_{12}}{u_{2}{ }^{\prime}}=0,297 ; & \beta_{2}{ }^{\prime \prime}=160^{0} 30^{\prime} .\end{cases}$

Nous voyons donc que l'inclinaison de l'aube sur le plan horizontal varie aussi d'une façon continue de l'intérieur. vers l'extérieur de la couronne. 
Enfin, en substituant dans l'équation (27) les valeurs de $B$, w et $A$, nous trouverons pour $\tilde{i}_{0}=\tilde{z}_{2}$ la valeur de l'angle du rayon vecteur d'un élément d'aube avec le rayon vecteur du point d'entrée dans la roue correspondant à cet élément d'aube sur la même trajectoire :

$$
\%=\frac{B}{2 !}\left(z_{1} \mathrm{~L} \frac{i}{i_{2}}-z_{1} \mathrm{~L} \frac{\tilde{i}_{1}}{\tau_{2}}-\tau_{0}+\tilde{i}_{1}\right)-\frac{\omega}{2 A} \mathrm{~L} \frac{i}{\tau_{1}},
$$

et par conséquent pour la sortie, c'est-à-dire pour $i=\tau_{2}$ :

$$
\%_{2}-=\frac{B}{2 \varphi}\left(z_{1} \mathrm{~L} \frac{z_{1}}{\tau_{2}}+z_{2}-z_{1}\right)-\frac{\omega}{2 A} \mathrm{~L} \frac{\tilde{i}_{2}}{i_{1}}
$$

soit, après calculs :

$$
\%_{2}=-\frac{0, \mathrm{r} 45}{\psi}-2,085
$$

et pour les bords extérieur et intérieur, soit pour "山’ et 'ل",

$$
\%^{\prime}=-1,763, \quad \%^{\prime \prime}=-0,970 .
$$

* Les longueurs d'arc correspondantes, comptécs à partir du plan méridien passant par la section d'entrée, comme l'indique la figure 11 , ont pour valeur:

$$
R_{2 \%}{ }^{\prime}=-\mathrm{I}^{\mathrm{m}} \mathrm{s}, \quad r_{2 \%}{ }^{\prime \prime}=-\mathrm{o}^{\mathrm{m} 3 \mathrm{~s}} .
$$

Le signe négatif indique que ces arcs doivent être mesurés dans le scns opposé au sens de rotation.

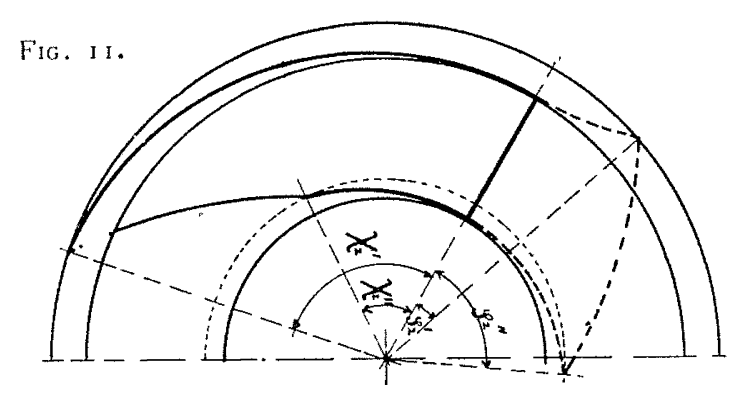

Les longueurs d'arc correspondantes pour la trajectoire absolue se dédursent de l'équation (26):

$$
p_{2}^{\prime}=-\frac{0,145}{\psi^{\prime}}=0,322, \quad \varphi_{2}^{\prime \prime}=-\frac{0,145}{\psi^{\prime \prime}}=1,115,
$$

et par conséquent ;

$$
R_{2} \varphi_{2}^{\prime}=\mathrm{om}_{2} 16, \quad r_{2} \tau_{2}^{\prime \prime}=\mathrm{o}^{\mathrm{m}_{4}} 4 .
$$

Ces longueurs sont reportées sur la figure II.

Nous avons ainsi déterminé tous les éléments nécessaires à la construction de notre turbine.

Nous ferons remarquer, d'ailleurs, que nous aurions pu adopter d'autres hypothèses, en particulier pour les valeurs limites de la composante tangentielle $w_{u}$, et que ces hypothèses auraient peut-être conduit à trouver des dimensions plus pratiques pour l'exécution. La figure in représente l'aube construite d'après les résultats de nos calculs, et, en pointillé, la surface des trajectoires absolues, dans l'hypothèse préliminaire que le bord d'entrée de l'aube est dirigé suivant un rayon.

\section{III. - Les turbines radiales.}

Nous allons maintenant étudier la turbıne radiale dont la forme est déterminée en coupe méridienne par les lignes d'écoulement indiquées à la figure 5, correspondant à la relation $(2 \mathrm{I})$. La roue de cẹtte turbine sera limitée entre deux rayons $r_{1}$ et $r_{2}$, comme le montre la figure i 2 . Si cette turbine radiale est une turbine centrifuge, les prolongements des parois limites de la roue mobile correspondent à l'extrémité de la conduite forcée, avec le distributeur; le plan origine des coordonnées sera situé immédiatement au. dessous de la roue. Si, au contrairc, cette turbine radiale

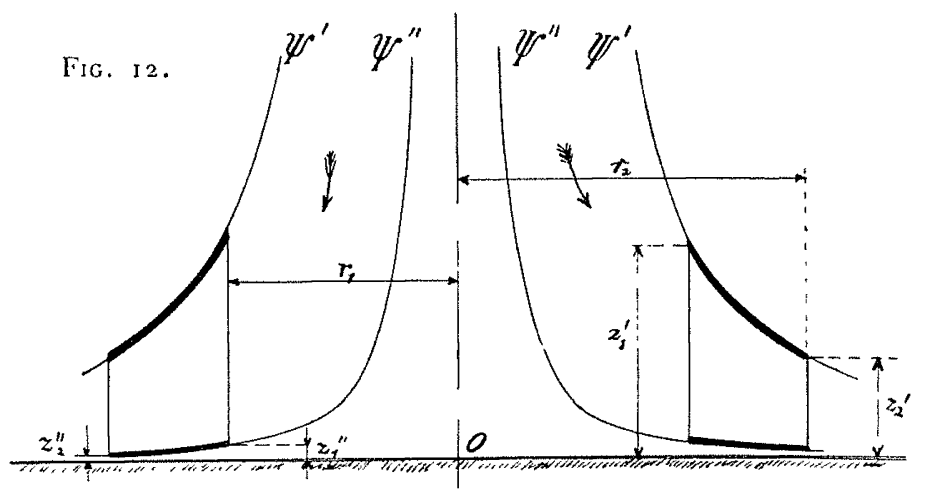

est centripète, il suffira de supposer toute la figure retournée. On sera tout naturellement amené, dans ce dernier cas, à placer après la roue, et à quelque distance de cette roue, un tuyau d'aspiration divergent vers le bas. Pour la turbine centrifuge, que seule nous envisagerons dans cette étude, l'emplacement rationnel de la turbine est dans le bief aval à une profondeur aussi grande que possible.

Désignons par $i_{1}$ et $z_{2}$ les ordonnées des points extrêmes du profil de la roue situés sur la ligne d'écoulement extérieure $\psi^{\prime}$; désignons par $z^{\prime \prime}{ }_{1}$ et $z^{\prime \prime}{ }_{2}$ les points correspondants sur la ligne intérieure $\psi^{\prime \prime}$. D’après les relations (2 I) et (23), le débit a pour expression :

$Q=-2 \pi \gamma A r_{1}^{2}\left(\tilde{i}_{1}^{\prime}-\tau_{1}^{\prime \prime}\right)=-2 \pi \gamma A r_{\psi}^{2}\left(z_{0}^{\prime}-z_{2}^{\prime \prime}\right)$,

soit, en tenant compte de la relation (22):

$Q=2 \pi w_{r_{1}} r_{1}\left(z_{1}^{\prime}-z_{1}^{\prime \prime}\right)=2 \pi_{r} w_{r_{2}} r_{2}\left(\tilde{i}^{\prime}-z_{2}^{\prime}\right)$.

Dans l'équation des moments (24), qui s'applique aussi dans le cas que nous étudions actuellement, il nous suffit de nous donner la valeur de la composante tangentielle $w_{u}$. $\mathrm{Si}$, par suite du caractère même de la turbine radiale, nous considérons cette composante comme n'étant fonction uniquement que de $r$, nous aurons, d'après la relation (24):

$$
\mathfrak{K}_{\mathcal{L}}=-2 \pi \frac{\gamma}{g} A \iint r^{2} \mathrm{~d} \tilde{z}^{\mathrm{d}}\left(w_{u} r\right),
$$

soit, en intégrant par rapport à $\tilde{\imath}$, c'est-à-dire sur une coupe verticale cylindrique :

$$
\mathfrak{x}=-2 \pi \frac{\gamma}{g} A \int r \cdot\left(\xi^{\prime}-\xi^{\prime \prime}\right) \mathrm{d}\left(w_{u} r\right) .
$$

Mais, d'après l'équation ( 31 ), nous avons :

$$
-2 \pi \gamma A r^{2}\left(\tilde{\imath}^{\prime}-\tau^{\prime \prime}\right)=Q=\text { constante }
$$

nous pouvons donc écrire l'équation $(32 a)$ en fạisant sortir du signe d'intégration le terme constant :

$$
\Re=\frac{Q}{g}\left(w_{u_{2}} r_{2}-w_{u_{1}} r_{1}\right)
$$

Cette équation est l'équation des moments d'Euler. Elle s'applique sans restriction aux turbines radiales, à la seule condition que la composante tangentielle soit uniquement 
fonction de la distance à l'axe de la particule liquide correspondante.

Désignons cette fonction par $\mathrm{f}(r)$. En divisant membre à membre les relations :

$$
\begin{aligned}
& r \frac{\mathrm{d}}{\mathrm{d} t}=w_{u}=\mathrm{f}(r), \\
& \frac{\mathrm{d} r}{\mathrm{~d} t}=w_{r}=-A r .
\end{aligned}
$$

nous aurons :

$$
\mathrm{d} \rho=-\frac{\mathrm{f}(r)}{A r^{2}} \mathrm{~d} r
$$

En intégrant cette relation, nous aurons l'équation de la trajectoire absolue en projection horizontale :

$$
\varphi=-\frac{\mathrm{I}}{A} \int_{r 1}^{r} \frac{\mathrm{f}(r)}{r^{2}} \mathrm{~d} r
$$

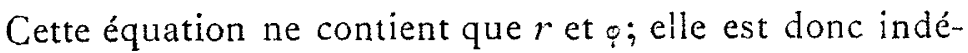
pendante de $\approx$. La même conclusion s'applique à la trajectoire relative, d'après la relation :

$$
\mathrm{d} \%=\mathrm{d} \varphi-\omega \mathrm{d} t=d \varphi-\omega \frac{\mathrm{d} r}{n^{\prime} r}
$$

soit, en nous reportant à la relation (33):

$$
\mathrm{d} \%=-\left(\frac{\mathrm{f}(r)}{A r^{2}}-\frac{\omega}{A r}\right) \mathrm{d} r
$$

et, après intégration :

$$
\%=-\frac{\mathrm{I}}{A}\left(\int_{r 1}^{r} \frac{\mathrm{f}(r)}{r_{2}} \mathrm{dr}-\omega_{\mathrm{L}} \frac{r_{1}}{r}\right)
$$

Supposons que l'aube, à l'entrée de l'eau dans la roue, ait une arète verticale; toutes les trajectoires relatives en projection horizontale, pour le même rayon initial $r$, seront semblables, et la surface de l'aube sera un cylindre vertical. Les autres éléments qui déterminent la forme de l'aube sont arbitraires, et c'est là la raison principale qui explique comment les turbines radiales s'adaptent facilement à des conditions de marche très différentes : il faut noter, en particulier, le fonctionnement des pompes centrifuges, qui ne sont, en somme, que des turbines centrifuges dont le sens de rotation est changé.

Dans une pompe centrifuge à aubes radiales, nous aurons :

$$
\mathrm{d} \%=\mathrm{o} \text {, }
$$

et la relation (34) devient :

$$
w_{u}=\mathrm{f}(r)=\omega r ;
$$

l'équation du moment $(32 b)$ se réduit à :

$$
\pi=\frac{Q}{g} \omega\left(r_{2}^{2}-r_{1}^{2}\right) \text {. }
$$

Posons d'une façon générale :

$$
w_{u}=B r
$$

l'équation (34), et par conséquent l'équation (34a) après intégration, devient :

$$
=\frac{\omega-B}{A} \mathrm{~L} \frac{r}{r_{1}}
$$

et la génératrice de l'aube est un spirale logarithmique. La théorie des turbines dites spiralordes se rattache donc tout naturellement à notre théorie. Il fáut remarquer, toutefors, que l'hypothèse correspondant à la relation (35) est incompatible avec celle qui suppose la composante tangentielle $w_{u}$ nulle à la sortie. Mais il suffira alors d'écrire:

$$
w_{u}=B\left(r-r_{2}\right)
$$

pour avoir, d'après la relation (34a):

$$
\%=\frac{\omega-B}{A} \mathrm{~L} \frac{r}{r_{1}}+\frac{B}{A} r_{2}\left(\frac{\mathrm{I}}{r_{1}}-\frac{1}{r}\right) .
$$

Le calcul même de la turbine se fait alors en déterminant d'abord la vitesse radiale de l'eau à l'entrée $w_{1}$, et la bauteur $z^{\prime}-z^{\prime \prime}$ de la section d'entrée; la relation :

$$
Q=2 \pi \gamma r_{1}\left(\ddot{u}^{\prime}-\tilde{u}^{\prime \prime}\right) u_{r_{1}}
$$

permet ensuite de calculer le rayon intérieur $r_{1}$. On déterminera de mème la vitesse radiale de l'eau à la sortie $w_{r a}$, et des formules :

$$
\frac{w_{r_{2}}}{w_{r_{1}}}-\frac{r_{2}}{r_{1}} \text { et }-A\left(r_{2}-r_{1}\right)=w_{r_{2}}-w_{1_{1}},
$$

on déduira les valeurs du rayon de sortie $r_{2}$ et de la constante $A$. En se donnant la hauteur $i$, on pourra calculer le paramètre u' de la fonction d'écoulement d'après la relation:

$$
\psi^{\prime}=A r_{1}^{2} \tilde{i}_{1},
$$

ét de même le paramètre $\bigcup^{\prime \prime}$, d'après la relation :

$$
2 \pi \gamma\left(\psi^{\prime}-\psi^{\prime \prime}\right)=Q \text {. }
$$

Enfin, en choisissant la valeur de l'angle $\alpha$ d'entrée, on pourra déduire la valeur de la composante tangentielle $w_{u_{1}}$ connaissant celle de $w_{r_{1}}$; puis, d'après la relation :

$$
w_{u_{1}}=B\left(r_{1}-r_{2}\right)
$$

on déterminera la valeur de la constante $B$.

Le moment de rotation est donné par la relation simple :

$$
\Re=-\frac{Q}{g} w_{u_{1}} r_{1} \text {. }
$$

Connaissant $\mathcal{H}$, on aura la valeur de la vitesse angulaire d'après la relation (3o):

$$
\omega=-\frac{r_{i} T}{\partial \mathfrak{K}}
$$

et les valeurs des vitesses tangentielles d'après les relations :

$$
u_{1}=r_{1} \omega, \quad u_{2}=r_{2} \omega .
$$

Enfin, de la relation (36), on déduira la forme de l'aube.

L'application numérique de cette théorie diffère très peu de celle traitée plus haut pour les turbines parallèles. Nous laisserons donc au lecteur le soin de la faire.

$$
\begin{aligned}
& \text { E. Kreitmanx, } \\
& \text { Ingénieur des Arts et Manufactures. } \\
& \text { G. RouTin, } \\
& \text { (A suive). }
\end{aligned}
$$

